\title{
Outcomes prediction in pre-operative radiotherapy locally advanced rectal cancer: leucocyte assessment as immune biomarker
}

\begin{abstract}
Alexis Vallard ${ }^{1}$, Max-Adrien Garcia ${ }^{2}$, Peng Diao ${ }^{3}$, Sophie Espenel ${ }^{1}$, Guy de Laroche $^{1}$, Jean-Baptiste Guy ${ }^{1}$, Majed Ben Mrad ${ }^{1}$, Chloé Rancoule ${ }^{1}$, David Kaczmarek ${ }^{4}$, Thierry Muron $^{5}$, Gregoire Pigné ${ }^{1}$, Jack Porcheron ${ }^{6}$, Michel Peoc'h7, Jean-Marc Phelip ${ }^{8}$, Julien Langrand-Escure ${ }^{1}$ and Nicolas Magné ${ }^{1}$

${ }^{1}$ Department of Radiation Oncology, Lucien Neuwirth Cancer Institute, Saint Priest en Jarez, France

${ }^{2}$ Public Health Department, Hygée Institute, Saint Priest en Jarez, France

${ }^{3}$ Department of Radiation Oncology, Sichuan Cancer Hospital, Chengdu, Sichuan Province, China

${ }^{4}$ Department of Thoracic and Digestive Surgery, Private Loire Hospital (HPL), Saint Etienne, France

${ }^{5}$ Department of Medical Oncology, Lucien Neuwirth Cancer Institute, Saint Priest en Jarez, France

${ }^{6}$ Department of Digestive and Oncologic Surgery, North University Hospital, Saint Priest en Jarez, France

${ }^{7}$ Department of Pathology, North University Hospital, Saint Priest en Jarez, France

${ }^{8}$ Department of Hepatology and Gastroenterology, North University Hospital, Saint Priest en Jarez, France

Correspondence to: Nicolas Magné, email: nicolas.magne@icloire.fr

Keywords: rectal cancer; chemoradiation; neutrophil; lymphocyte; ratio

Received: September 01, $2017 \quad$ Accepted: March 19, $2018 \quad$ Published: April 27, 2018

Copyright: Vallard et al. This is an open-access article distributed under the terms of the Creative Commons Attribution License 3.0 (CC BY 3.0), which permits unrestricted use, distribution, and reproduction in any medium, provided the original author and source are credited.
\end{abstract}

\section{ABSTRACT}

Objective: Leukocytes are hypothesized to reflect the inflammatory tumor microenvironment. We aimed to validate their prognostic significance in a large cohort of patients treated with pre-operative radiation for locally advanced rectal cancer (RC).

Results: From 2004 to 2015, 257 RC patients with available biological data underwent a pre-operative radiotherapy, with a median age of 66 years. The median rectal EQD2 was 49.2Gy. Most of patients experienced concurrent chemotherapy $(n=245,95.4 \%)$, mainly with $5-F U(83.3 \%)$. Clear surgical margins (i.e. complete resection) were achieved in 234 patients (91.1\%). A complete (Mandard TRG1: $n=35,13.6 \%$ ) or almost complete pathological response (Mandard TRG2: $n=$ $56,21.8 \%$ ) were achieved in 91 patients (35.4\%). With a median follow-up of 46.1 months, 8 patients $(3.1 \%)$ experienced local relapse, $38(14.8 \%)$ experienced metastases and $45(17.5 \%)$ died. Elevated pre-radiation neutrophil to lymphocyte ratio (NLR > 2.8) was identified as an independent predictive factor of increased local relapse, of decreased progression-free survival and overall survival in multivariate analysis. Elevated NLR was marginally associated with incomplete pathological response in multivariate analysis, suggesting a possible value as a biomarker of radio-sensitivity.

Conclusions: Pre-radiation NLR is a simple and robust biomarker for risk stratification in locally advanced RC patients undergoing pre-operative radiotherapy, and might select the subpopulation eligible to treatment intensification or to neoadjuvant chemotherapy. 


\section{Material and Methods: Clinical records from consecutive patients treated in a single institution between 2004 and 2015 with curative-intent radiotherapy were retrospectively analyzed. Classical prognosis factors of RC and peripheral immune markers based on lymphocytes and neutrophil counts were studied.}

\section{INTRODUCTION}

In a recent meta-analysis, the 7-year overall survival (OS) rate of stage II/III locally advanced rectal cancer (RC) patients was $62 \%$ despite optimization of the treatment planning process and integration of systemic agents to improve treatment efficacy [1]. Currently identified prognosis factors for survival ( $\mathrm{T}$ stage, age, grade, number of examined lymph nodes and number of positive nodes [2]) are therefore not discriminant enough to detect patients with the highest risks of tumor relapse. Identification of new biomarkers is crucial in order to isolate the subpopulation that might require treatment intensification. Unlike promising but expensive biomarkers based on genetic profiling [3, 4], leukocyte and neutrophil counts are already performed in daily routine, making them accessible regardless of the local technological development. Interestingly, there is a strong rationale suggesting that systemic inflammatory response to tumors is manifested in the peripheral white blood cells [5-7]. In various primary tumor locations, peripheral immune markers (leukocytosis, neutrophilia, neutrophil to lymphocyte ratio (NLR)) were recently suggested to be possible biomarkers of radiosensitivity, predicting local relapse and survival in patients treated with definitive chemoradiation $[5,7,8]$. The NLR was suggested to be an independent prognostic factor for recurrence and mortality in large cohorts of colorectal cancer patients $[9,10]$ and in small cohorts of RC patients [11-15]. However, to our best knowledge, the impact of peripheral immune markers on prognostication has never been assessed specifically in a large cohort of locally advanced RC patients.

The aim of the present study was to analyze whether peripheral immune markers of locally advanced $\mathrm{RC}$ patients was associated with local tumor control and global outcome.

\section{RESULTS}

\section{Patient, tumor and treatment characteristics}

A total of 477 consecutive metastasis-free RC patients underwent a pre-operative radiotherapy between 2004 and 2015 at the institute, with 220 exclusions because of missing biological data. Thus, data of 257 patients were analyzed in the present study. At time of radiotherapy, median age was 66 years (IQR: 56.4-73), with 170 males and 87 females. Patients were in good condition, with 251 patients $(97.7 \%)$ with an WHO Performance Status $0-1$. Most of RCs $(n=239,93 \%)$ were diagnosed at a locally advanced setting (stage IIIII). Tumors were located in the lower $(n=114,44.4 \%)$, middle $(n=117,45.5 \%)$ or upper $(n=26,10.1 \%)$ rectum. Pathological analyses identified adenocarcinoma $(n=257,100 \%)$, mainly with moderate differentiation $(n=126,49 \%)$. Vascular invasion was identified in 56 (20.2\%) tumors. The baseline median lymphocyte count was $1.81 \times 10^{9} / \mathrm{L}$ (IQR: $1.45-2.21$ ). The baseline median neutrophil count was $4.05 \times 10^{9} / \mathrm{L}$ (IQR: $3.17-5.35$ ). There was no statistical correlation between the baseline median neutrophil count and the tumor stage $(p=0.06)$. All patients underwent a preoperative radiotherapy, mainly based on 3D-conformational radiotherapy $(n=243$, $94.5 \%)$, without rectal dose reduction $(n=130,50.6 \%)$. Median rectal EQD2 was 49.2 Gy (IQR = 43.9-50), with a median radiation duration of 5 weeks (IQR: 4.6-5.3). The median dose per fraction was 2.0 Gy (IQR: 1.8-2.0). Median time from radiotherapy completion to surgery was 6.6 weeks (IQR: 5.9-7.3). Chemotherapy was concurrently associated with radiotherapy in most of patients $(n=$ $245,95.3 \%)$. Oral or intravenous 5-FU was mainly used $(n=214,83.3 \%)$. The median follow-up was 3.8 years (IQR: 1.9-5.4). Patient, tumor and treatment characteristics are reported in Table 1.

\section{Adjuvant treatments}

An adjuvant chemotherapy was performed in 100 patients (38.9\%), depending on pre-operative imaging and on pathological findings. Thus, 40 patients $(15.5 \%)$ with post-operative node involvement on pathological findings underwent adjuvant chemotherapy. Furthermore, 60 patients $(23.3 \%)$ with node involvement on pre-operative imaging (MRI, TDM, US) but without post-operative node involvement (i.e. complete pathological response on lymph nodes) underwent adjuvant chemotherapy. A total of 28 patients $(10.9 \%)$ with a theoretical indication of chemotherapy (node involvement) on pathological findings were recused, either because of age, immediate distant progression (metastases) or because of post-operative complications (fistulae).

Oxaliplatin plus infusional 5-fluorouracil (5-FU) and folinic acid (FOLFOX, $n=64,24.9 \%$ ) or exclusive 5-FU $(n=33,12.8 \%)$ were mainly prescribed. Interestingly, only 33 FOLFOX patients (53.1\%) and 23 exclusive 5-FU patients $(69.7 \%)$ received the full dose adjuvant chemotherapy. 
Table 1: Patient, tumor and treatment characteristics

\begin{tabular}{|c|c|c|c|c|}
\hline Variables & $\begin{array}{c}\text { Whole set of } \\
\text { patients } \\
(n=257,100 \%)\end{array}$ & $\begin{array}{c}\text { Patients with } \\
\text { pre-radiation } \\
\text { NTL } \leq 2.8 \\
(n=169,65.6 \%)\end{array}$ & $\begin{array}{c}\text { Patients with } \\
\text { pre-radiation } \\
\text { NTL }>2.8 \\
(n=71,27.6 \%)\end{array}$ & $p$ value \\
\hline \multicolumn{5}{|l|}{ Clinical data } \\
\hline Median age, years (IQR) & $66.0(56.4-73.0)$ & $66.8(56.9-72.7)$ & $63.1(53.6-75.6)$ & $0.39^{\dagger}$ \\
\hline$<70$ years, $n(\%)$ & $173(67.3)$ & $114(67.5)$ & $50(70.4)$ & $0.77^{\gamma}$ \\
\hline$\geq 70$ years, $n(\%)$ & $84(32.7)$ & $55(32.5)$ & $21(29.6)$ & \\
\hline Gender, $n(\%)$ & & & & $0.28^{\gamma}$ \\
\hline Male & $170(66.1)$ & $110(65.1)$ & $52(73.2)$ & \\
\hline Female & $87(33.9)$ & $59(34.9)$ & $19(26.8)$ & \\
\hline WHO Performance status, $n(\%)$ & & & & $1^{*}$ \\
\hline $0-1$ & $251(97.7)$ & $167(98.8)$ & $70(98.6)$ & \\
\hline $2-3$ & $6(2.3)$ & $2(1.2)$ & $1(1.4)$ & \\
\hline Body mass index, $n(\%)$ & & & & 0.06 \\
\hline$<18.5$ & $6(2.3)$ & $2(1.18)$ & $4(5.6)$ & \\
\hline$\geq 18.5$ & $219(85.2)$ & $147(86.98)$ & $57(80.3)$ & \\
\hline Missing data & 32 & $20(11.8)$ & $10(14.1)$ & \\
\hline Median follow-up, months (IQR) & $46.1(23.3-64.7)$ & $52.4(28.2-66.4)$ & $35.9(17.9-60.6)$ & $0.02^{\dagger}$ \\
\hline \multicolumn{5}{|l|}{ Biological data $(\mathrm{G} / \mathrm{L})$} \\
\hline \multicolumn{5}{|l|}{ Pre-radiation } \\
\hline Median neutrophil count & $4.05(3.17-5.35)$ & $3.60(2.93,4.50)$ & $5.48(4.59-6.71)$ & $<0.0011^{\dagger}$ \\
\hline Median lymphocyte count & $1.81(1.45-2.21)$ & $2.03(1.69-2.35)$ & $1.48(1.27-1.7)$ & $<0.0011^{\dagger}$ \\
\hline \multicolumn{5}{|l|}{4 weeks after radiation initiation } \\
\hline Median neutrophil count & $3.00(2.31-3.99)$ & $2.71(2.18-3.45)$ & $3.70(3.00-4.90)$ & $<0.001^{\dagger}$ \\
\hline Median lymphocyte count & $0.70(0.55-0.90)$ & $0.76(0.6-0.97)$ & $0.60(0.47-0.77)$ & $<0.001+$ \\
\hline \multicolumn{5}{|l|}{ Tumor data, $n(\%)$} \\
\hline Location & & & & $0.81^{\gamma}$ \\
\hline Lower rectum & $114(44.4)$ & $74(43.8)$ & $32(45.0)$ & \\
\hline Middle rectum & $117(45.5)$ & $76(45.0)$ & $33(46.5)$ & \\
\hline Upper rectum & $26(10.1)$ & $19(11.2)$ & $6(8.5)$ & \\
\hline \multicolumn{5}{|l|}{ Tumor Histology } \\
\hline Adenocarcinoma & $257(100)$ & 169 & 71 & \\
\hline Tumor differenciation & & & & $0.001^{\ddagger}$ \\
\hline Poor & $9(3.5)$ & $4(2.4)$ & $5(7.0)$ & \\
\hline Moderate & $126(49.0)$ & $76(45.0)$ & $43(60.6)$ & \\
\hline Well & $108(42.0)$ & $82(48.5)$ & $17(23.9)$ & \\
\hline Missing data & $14(5.5)$ & $7(4.1)$ & $6(8.5)$ & \\
\hline Tumor staging & & & & $0.65^{*}$ \\
\hline Stage I & $12(4.7)$ & $7(4.1)$ & $4(5.6)$ & \\
\hline Stage II & $79(30.7)$ & $56(33.1)$ & $20(28.2)$ & \\
\hline Stage III & $160(62.3)$ & $102(60.4)$ & $46(64.8)$ & \\
\hline
\end{tabular}




\begin{tabular}{|c|c|c|c|c|}
\hline Unknown (Tx Nx M0) & $6(2.3)$ & $4(2.4)$ & $1(1.4)$ & \\
\hline Vascular invasion & & & & $0.42^{\gamma}$ \\
\hline Yes & $56(21.8)$ & $33(19.5)$ & $17(24.0)$ & \\
\hline No & $181(70.4)$ & $125(74.0)$ & $46(64.8)$ & \\
\hline Missing data & $20(7.8)$ & $11(6.5)$ & $8(11.2)$ & \\
\hline \multicolumn{5}{|l|}{ Radiation data } \\
\hline Median rectal EQD2, Gy (IQR) & $49.2(43.9-50.0)$ & $49.2(43.9-50.0)$ & $49.2(47.7-50.0)$ & $0.26^{\dagger}$ \\
\hline Median dose per fraction, Gy (IQR) & $2.0(1.8-2.0)$ & $2.0(1.80-2.0)$ & $2.0(1.8-2.0)$ & $0.72^{\dagger}$ \\
\hline Median duration, days (IQR) & $35(32-36)$ & $35(32-36)$ & $35(32-37)$ & $0.35^{\dagger}$ \\
\hline Radiation technique, $n(\%)$ & & & & $0.83^{\gamma}$ \\
\hline 3D-CRT & $243(94.5)$ & $160(94.7)$ & $66(93.0)$ & \\
\hline IMRT & $14(5.5)$ & $9(5.3)$ & $5(7.0)$ & \\
\hline Normofractionated regimen, $n(\%)$ & $237(92.2)$ & $159(94.1)$ & $65(91.5)$ & $0.66^{\gamma}$ \\
\hline Hypofractionated regimen, $n(\%)$ & $20(7.8)$ & $10(5.9)$ & $6(8.5)$ & \\
\hline Rectal dose reduction, $n(\%)$ & & & & $0.33^{\gamma}$ \\
\hline Yes & $127(49.4)$ & $82(48.5)$ & $40(56.4)$ & \\
\hline No & $130(50.6)$ & $87(51.5)$ & $31(43.6)$ & \\
\hline $\begin{array}{l}\text { Time from radiation completion to } \\
\text { surgery, days }\end{array}$ & $46(41-51)$ & $46(41-50)$ & $47(43-54)$ & $0.15^{\dagger}$ \\
\hline \multicolumn{5}{|l|}{ Chemotherapy data, $n(\%)$} \\
\hline Concomitant chemotherapy & $245(95.4)$ & $164(97)$ & $67(94.4)$ & $1^{*}$ \\
\hline 5-FU & $214(83.3)$ & $143(84.6)$ & $59(83.1)$ & \\
\hline FOLFOX & $19(7.4)$ & $13(7.7)$ & $5(7.1)$ & \\
\hline Other & $12(4.7)$ & $8(4.7)$ & $3(4.2)$ & \\
\hline No chemotherapy & $12(4.6)$ & $5(3.0)$ & $4(5.6)$ & \\
\hline
\end{tabular}

Percentages were calculated based on the population of each column.

Abbreviations: IQR: interquartile range, n: number of patients, 3D-CRT: 3D conformational radiotherapy; IMRT: Intensitymodulated radiotherapy; ${ }^{\dagger}$ Kruskal-Wallis test, ${ }^{\dagger}$ Fischer test; ${ }^{\gamma}$ Khi-square test.

\section{Outcomes}

\section{Complete resection}

Clear surgical margins (i.e. complete resection) were achieved in 234 patients (91.1\%). A logistic regression was performed in order to assess factors associated with complete resection. The univariate analysis provided potential $(p<0.20)$ predictive factors, with WHO performance status $0-1(\mathrm{HR}=10.45 \mathrm{CI} 95 \%(1.35-60.06))$ and concomitant chemotherapy (HR $=4.54$ CI 95\% $(0.64-20.51), p=0.07)$. Potential $(p<0.20)$ risk factors of incomplete resection were age $\geq 70$ (HR $=0.39$ CI $95 \%$ $(0.12-1.21), p=0.09)$, vascular invasion $(\mathrm{HR}=0.14 \mathrm{CI}$ $95 \%(0.03-0.45), p=0.001)$, and time interval between radiation completion and surgery $\geq 56$ days $(\mathrm{HR}=0.34 \mathrm{CI}$ $95 \%(0.10-1.32), p=0.09)$. In multivariate analysis, only the vascular invasion was an independent predictive factor of incomplete resection ( $\mathrm{HR}=0.15 \mathrm{CI} 95 \%(0.04-0.52)$, $p=0.003)$. Uni- and multi-variate analysis is reported in Table 2.

\section{Pathological response}

A complete (Mandard TRG1: $n=35,13.6 \%$ ) or almost complete pathological response (Mandard TRG2: $n$ $=56,21.8 \%$ ) was achieved in 91 patients (35.4\%). A logistic regression was performed in order to assess the associated factors with good pathological response (Mandard TRG1$2)$. The univariate analysis provided potential $(p<0.20)$ predictive factors of good response, with WHO performance status $0-1(\mathrm{HR}=4.63 \mathrm{CI} 95 \%(1.06-20.35), p=0.04)$ concurrent chemotherapy $(\mathrm{HR}=2.22 \mathrm{CI} 95 \%(1.34-3.61)$, $p=0.001)$, rectal EQD2 $\geq 45$ Gy $(\mathrm{HR}=1.41 \mathrm{CI} 95 \%(0.9-$ $2.22), p=0.13)$, pelvic $\mathrm{EQD} 2 \geq 40 \mathrm{~Gy}(\mathrm{HR}=2.37 \mathrm{CI} 95 \%$ $(1.05-5.27), p=0.02)$, and 4WPRN ratio $>1.1(\mathrm{HR}=2.03$ CI 95\% (0.87-4.74), $p=0.10)$. Potential $(p<0.20)$ predictive factors of bad response were age $\geq 70$ ( $\mathrm{HR}=0.49$ CI $95 \%$ 
Table 2: Prognostic factors for complete resection (clear surgical margins) based on univariate and multivariate analysis ( $n=277$ patients with 234 complete resections, 13 involved surgical margins, 10 NA)

\begin{tabular}{|c|c|c|c|c|c|}
\hline \multirow[b]{2}{*}{ Variable } & \multicolumn{3}{|c|}{ Univariate analysis } & \multicolumn{2}{|c|}{ Multivariate analysis } \\
\hline & $\begin{array}{l}\text { Tested } v s . \text { Adverse } \\
\text { criteria }\end{array}$ & $\begin{array}{c}\text { Logistic regression } \\
\text { coefficient } \\
(95 \% \mathrm{CI})\end{array}$ & $p$-value & $\begin{array}{c}\text { Logistic regression } \\
\text { coefficient } \\
(95 \% \mathrm{CI})\end{array}$ & $p$-value \\
\hline Age & $\geq 70$ vs. $<70$ & $0.39(0.12-1.21)$ & 0.09 & $0.39(0.11-1.39)$ & 0.15 \\
\hline Gender & Female $v s$. Male & $0.56(0.18-1.80)$ & 0.31 & & \\
\hline $\begin{array}{l}\text { WHO performance } \\
\text { status }\end{array}$ & $0-1$ vs. $2-3$ & $10.45(1.35-60.06)$ & 0.01 & $6.31(0.69-45)$ & 0.07 \\
\hline Body Mass Index & $\geq 18.5$ vs. $<18.5$ & N.A. & 0.99 & & \\
\hline Tumor stage & Stage III $v s$. I-II & $0.85(0.22-2.77)$ & 0.79 & & \\
\hline \multirow[t]{2}{*}{ Rectal tumor location } & Upper $v s$. Middle & $0.90(0.13-17.97)$ & 0.92 & & \\
\hline & Lower vs. Middle & $0.48(0.13-1.58)$ & 0.24 & & \\
\hline \multirow[t]{2}{*}{ Tumor differentiation } & Poor vs. Well & N.A. & 0.99 & & \\
\hline & Moderate vs. Well & $0.82(0.23-56.11)$ & 0.73 & & \\
\hline ypCR & Yes vs. No & N.A. & 0.99 & & \\
\hline Vascular invasion & Yes vs. No & $0.14(0.03-0.45)$ & 0.001 & $0.15(0.04-0.52)$ & 0.003 \\
\hline $\begin{array}{l}\text { Time interval between } \\
\text { radiation completion } \\
\text { and tumor resection }\end{array}$ & $\geq 56$ days vs. $<56$ days & $0.34(0.10-1.32)$ & 0.09 & & \\
\hline Pre-Radiation NLR & $>2.8 v s . \leq 2.8$ & $1.27(0.27-4.59)$ & 0.74 & & \\
\hline 4WPRN ratio & $>1.1 v s . \leq 1.1$ & $1.04(0.17-19.82)$ & 0.97 & & \\
\hline 4WPRL ratio & $>0.35 v s . \leq 0.35$ & $1.14(0.23-4.81)$ & 0.86 & & \\
\hline 4WPL ratio & $>2.5 v s . \leq 2.5$ & $2.09(0.44-7.69)$ & 0.30 & & \\
\hline \multirow[t]{3}{*}{$\begin{array}{l}\text { Radiotherapy } \\
\text { characteristics }\end{array}$} & $\begin{array}{l}\text { Rectal EQD2 } \geq 45 \text { Gy } v s \text {. } \\
<45 \text { Gy }\end{array}$ & $1.16(0.30-3.68)$ & 0.82 & & \\
\hline & $\begin{array}{l}\text { Pelvis EQD2 } \geq 40 \text { Gy } v s \text {. } \\
<40 \text { Gy }\end{array}$ & $2.35(0.12-14.4)$ & 0.44 & & \\
\hline & $\begin{array}{l}\text { Hypofractionated } v s . \\
\text { Normofractionated }\end{array}$ & $0.46(0.11-3.10)$ & 0.34 & & \\
\hline $\begin{array}{l}\text { Concomitant } \\
\text { chemotherapy }\end{array}$ & Yes vs. No & $4.54(0.64-20.51)$ & 0.07 & & \\
\hline
\end{tabular}

95\% CI: 95\% confidence interval; ypCR: Pathological complete response (Mandard TRG1); NLR: Neutrophil to Lymphocyte ratio; 4WPRN ratio: "4 weeks" to "pre-radiation" neutrophil ratio; 4WPRL ratio: "4 weeks" to "pre-radiation" lymphocyte ratio; 4WPL ratio: "4 weeks" neutrophil to "4 weeks" lymphocyte ratio. N.A.: Not assessable (no event), typically giving infinity values. EQD2: Equivalent Dose in 2 Grays per fraction; Normofractionated: $<2.5$ Gy per fraction; Hypofractionated: $\geq 2.5$ Gy per fraction.

All $p$-values $\leq 0.2$ in univariate analysis have been tested in multivariate analysis, except correlated variables (correlation with $p<0.001)$. Finally, only bold typed values were tested in multivariate analysis.

$(0.33-0.74), p<0.001)$, stage III tumor (HR $=0.61$ CI $95 \%$ $(0.41-0.92), p=0.02)$, hypofractionation ( $\mathrm{HR}=0.51 \mathrm{CI} 95 \%$ $(0.24-1.1), p=0.07$ ) and pre-radiation NLR $>2.8$ (HR $=0.54 \mathrm{CI} 95 \%(0.29-1.01), p=0.05)$. In multivariate analysis, age $\geq 70$ years ( $\mathrm{HR}=0.42 \mathrm{CI} 95 \%(0.22-0.79)$, $p=0.08)$ and tumor stage III $(\mathrm{HR}=0.44(0.25-0.80)$, $p=0.006)$ were identified as independent risk factor of poor pathological response. Pre-radiation NTL ratio $>2.8$ was associated with a close-to-significant higher risk of poor pathological response $(\mathrm{HR}=0.53 \mathrm{CI} 95 \%(0.27-1.02)$, $p=0.06)$. Uni- and multi-variate analysis is reported in Table 3. 
Table 3: Prognostic factors for good pathological response (Mandard TRG1-2) based on univariate and multivariate analysis $(n=257$ patients with 35 Mandard TRG1 and 56 Mandard TRG2)

\begin{tabular}{|c|c|c|c|c|c|}
\hline \multirow[b]{2}{*}{ Variable } & \multicolumn{3}{|c|}{ Univariate analysis } & \multicolumn{2}{|c|}{ Multivariate analysis } \\
\hline & $\begin{array}{l}\text { Tested vs. Adverse } \\
\text { criteria }\end{array}$ & $\begin{array}{c}\text { Logistic regression } \\
\text { coefficient } \\
(95 \% \mathrm{CI})\end{array}$ & $p$-value & $\begin{array}{c}\text { Logistic regression } \\
\text { coefficient } \\
(95 \% \mathrm{CI})\end{array}$ & $p$-value \\
\hline Age & $\geq 70 v s .<70$ & $0.49(0.33-0.74)$ & $<0.001$ & $0.42(0.22-0.79)$ & 0.008 \\
\hline Gender & Female vs. Male & $0.92(0.62-1.39)$ & 0.7 & & \\
\hline $\begin{array}{l}\text { WHO performance } \\
\text { status }\end{array}$ & $0-1$ vs. 2-3 & $4.63(1.05-20.35)$ & 0.04 & & \\
\hline Body mass index & $\geq 18.5$ vs. $<18.5$ & $2.25(0.62-8.12)$ & 0.19 & & \\
\hline Tumor stage & Stage III vs. I-II & $0.61(0.41-0.92)$ & 0.02 & $0.44(0.25-0.80)$ & 0.006 \\
\hline \multirow[t]{2}{*}{ Rectal tumor location } & Upper $v s$. Middle & $0.86(0.49-1.87)$ & 0.90 & & \\
\hline & Lower vs. Middle & $0.93(0.62-1.42)$ & 0.75 & & \\
\hline \multirow[t]{2}{*}{ Tumor differentiation } & Poor vs. Well & $0.68(0.25-1.83)$ & 0.45 & & \\
\hline & Moderate $v s$. Well & $0.85(0.55-1.30)$ & 0.44 & & \\
\hline $\begin{array}{l}\text { Time interval } \\
\text { between radiation } \\
\text { completion and tumor } \\
\text { resection }\end{array}$ & $\geq 56$ days $v s .<56$ days & $0.91(0.55-1.52)$ & 0.73 & & \\
\hline Pre-Radiation NLR & $>2.8 v s . \leq 2.8$ & $0.54(0.29-1.01)$ & 0.05 & $0.53(0.27-1.02)$ & 0.06 \\
\hline 4WPRN ratio & $>1.1$ vs. $\leq 1.1$ & $2.03(0.87-4.74)$ & 0.10 & & \\
\hline 4WPRL ratio & $>0.35 v s . \leq 0.35$ & $0.68(0.37-1.24)$ & 0.21 & & \\
\hline 4WPL ratio & $>2.5 v s . \leq 2.5$ & $1.51(0.67-3.38)$ & 0.32 & & \\
\hline \multirow[t]{3}{*}{$\begin{array}{l}\text { Radiotherapy } \\
\text { characteristics }\end{array}$} & $\begin{array}{l}\text { Rectal EQD2 } \geq 45 \text { Gy } \\
\text { vs. }<45 \mathrm{~Gy}\end{array}$ & $1.41(0.9-2.22)$ & 0.13 & $1.62(0.83-3.16)$ & 0.16 \\
\hline & $\begin{array}{l}\text { Pelvis EQD2 } \geq 40 \mathrm{~Gy} \\
v s .<40 \mathrm{~Gy}\end{array}$ & $2.37(1.05-5.27)$ & 0.02 & & \\
\hline & $\begin{array}{l}\text { Hypofractionated } v s \text {. } \\
\text { Normofractionated }\end{array}$ & $0.51(0.24-1.1)$ & 0.07 & & \\
\hline $\begin{array}{l}\text { Concomitant } \\
\text { chemotherapy }\end{array}$ & Yes vs. No & $2.22(1.34-3.61)$ & 0.001 & & \\
\hline
\end{tabular}

95\% CI: 95\% confidence interval; ypCR: Pathological complete response (Mandard TRG1); NLR: Neutrophil to Lymphocyte ratio; 4WPRN ratio: "4 weeks" to "pre-radiation" neutrophil ratio; 4WPRL ratio: "4 weeks" to "pre-radiation" lymphocyte ratio; 4WPL ratio: "4 weeks" neutrophil to "4 weeks" lymphocyte ratio. N.A.: Not assessable (no event), typically giving infinity values. EQD2: Equivalent Dose in 2 Grays per fraction; Normofractionated: $<2.5$ Gy per fraction; Hypofractionated: $\geq 2.5$ Gy per fraction.

All $p$-values $\leq 0.2$ in univariate analysis have been tested in multivariate analysis, except correlated variables (correlation with $p<0.001)$. Finally, only bold typed values were tested in multivariate analysis.

\section{Local recurrence}

At the end of follow-up, 8 patients (3.1\%) experienced local relapse after radiotherapy in the whole set of patients. A logistic regression was performed in order to assess the associated factors with local recurrence. The univariate analysis provided potential $(p<0.20)$ predictive protective factors, with complete tumor resection
$(\mathrm{HR}=0.05 \mathrm{CI} 95 \%(0.01-0.29), p<0.001)$ and tumor moderate differentiation $(\mathrm{HR}=0.04 \mathrm{CI} 95 \%(0.05-1.57)$, $p=0.19)$. Potential $(p<0.20)$ predictive risk factors were also identified with pre-radiation NLR $>2.8(\mathrm{HR}=4.98$ CI 95\% (0.95-36.5), $p=0.07)$. In multivariate analysis, complete tumor resection $(\mathrm{HR}=0.05 \mathrm{CI} 95 \%(0.01-0.29)$, $p<0.001)$ and well differentiated tumors ( $\mathrm{HR}=0.04$ $(0.01-0.5), p=0.04)$ were identified as independent 
Table 4: Prognostic factors for local tumor recurrence based on univariate and multivariate analysis $(n=257$ patients with 8 local recurrences)

\begin{tabular}{|c|c|c|c|c|c|}
\hline \multirow[b]{2}{*}{ Variable } & \multicolumn{3}{|c|}{ Univariate analysis } & \multicolumn{2}{|c|}{ Multivariate analysis } \\
\hline & $\begin{array}{c}\text { Tested } v s . \text { Adverse } \\
\text { criteria }\end{array}$ & $\begin{array}{c}\text { Logistic regression } \\
\text { coefficient } \\
(95 \% \mathrm{CI})\end{array}$ & $p$-value & $\begin{array}{c}\text { Logistic regression } \\
\text { coefficient } \\
(95 \% \mathrm{CI})\end{array}$ & $p$-value \\
\hline Age & $\geq 70 v s .<70$ & $2.11(0.49-9.14)$ & 0.29 & & \\
\hline Gender & Female $v s$. Male & $2.0(0.46-8.65)$ & 0.34 & & \\
\hline WHO performance status & $0-1$ vs. 2-3 & N.A. & N.A. & & \\
\hline Body mass index & $\geq 18.5$ vs. $<18.5$ & N.A. & 0.99 & & \\
\hline Tumor stage & Stage III vs. I-II & $0.95(0.23-4.70)$ & 0.94 & & \\
\hline \multirow[t]{2}{*}{ Rectal tumor location } & Upper vs. Middle & N.A. & 0.99 & & \\
\hline & Lower vs. Middle & $1.74(0.42-8.66)$ & 0.45 & & \\
\hline \multirow[t]{2}{*}{ Tumor differentiation } & Well vs. Poor & N.A. & 0.99 & N.A. & 0.99 \\
\hline & Well vs. Moderate & $0.33(0.05-1.57)$ & 0.19 & $0.04(0.001-0.50)$ & 0.04 \\
\hline $\begin{array}{l}\text { Complete tumor } \\
\text { resection }\end{array}$ & Yes vs. No & $0.07(0.02-0.39)$ & 0.001 & $0.02(0.001-0.27)$ & 0.004 \\
\hline ypCR & Yes vs. No & $0.98(0.05-6.0)$ & 0.97 & & \\
\hline Vascular invasion & Yes vs. No & $1.08(0.16-4.85)$ & 0.93 & & \\
\hline $\begin{array}{l}\text { Time interval between } \\
\text { radiation completion and } \\
\text { tumor resection }\end{array}$ & $\geq 56$ days $v s .<56$ days & N.A. & 0.93 & & \\
\hline Pre-Radiation NLR & $>2.8 v s . \leq 2.8$ & $4.98(0.95-36.5)$ & 0.07 & $14.7(1.53-334.30)$ & 0.03 \\
\hline 4WPRN ratio & $>1.1$ vs. $\leq 1.1$ & N.A. & 0.99 & & \\
\hline 4WPRL ratio & $>0.35$ vs. $\leq 0.35$ & $0.539(0.06-4.57)$ & 0.54 & & \\
\hline 4WPL ratio & $>2.5$ vs. $\leq 2.5$ & N.A. & 0.99 & & \\
\hline \multirow[t]{3}{*}{$\begin{array}{l}\text { Radiotherapy } \\
\text { characteristics }\end{array}$} & $\begin{array}{l}\text { Rectal EQD2 } \geq 45 \mathrm{~Gy} \\
v s .<45 \mathrm{~Gy}\end{array}$ & $0.61(0.15-3.05)$ & 0.51 & & \\
\hline & $\begin{array}{l}\text { Pelvis EQD2 } \geq 40 \mathrm{~Gy} \\
\text { vs. }<40 \mathrm{~Gy}\end{array}$ & N.A. & 0.99 & & \\
\hline & $\begin{array}{l}\text { Hypofractionated } v s . \\
\text { Normofractionated }\end{array}$ & N.A. & 0.99 & & \\
\hline $\begin{array}{l}\text { Concomitant } \\
\text { chemotherapy }\end{array}$ & Yes vs. No & $0.32(0.05-6.32)$ & 0.31 & & \\
\hline
\end{tabular}

95\% CI: 95\% confidence interval; ypCR: Pathological complete response (Mandard TRG1); NLR: Neutrophil to Lymphocyte ratio; 4WPRN ratio: "4 weeks" to "pre-radiation" neutrophil ratio; 4WPRL ratio: "4 weeks" to "pre-radiation" lymphocyte ratio; 4WPL ratio: "4 weeks" neutrophil to "4 weeks" lymphocyte ratio. N.A.: Not assessable (no event), typically giving infinity values. EQD2: Equivalent Dose in 2 Grays per fraction; Normofractionated: $<2.5$ Gy per fraction; Hypofractionated: $\geq 2.5$ Gy per fraction.

All $p$-values $\leq 0.2$ in univariate analysis have been tested in multivariate analysis, except correlated variables (correlation with $p<0.001$ ). Finally, only bold typed values were tested in multivariate analysis.

protective factors of local recurrence. Pre-radiation NLR $>2.8$ was identified as an independent risk factor of local recurrence $(\mathrm{HR}=14.7 \mathrm{CI} 95 \%(1.53-334.3), p=0.03)$. Uni- and multi-variate analysis is reported in Table 4.

\section{Progression-free survival (PFS)}

At last follow-up, 8 patients (3.1\%) experienced local relapse, 38 (14.8\%) experienced metastases and 45 
(17.5\%) had died. Median PFS was therefore not reached. Two-year and 5-year PFS were 79.4\% (CI95\%: 74.384.9) and 67.7\% (CI 95\%: 61.3-74.3), respectively. A logistic regression (univariate and multivariate analysis) was performed in order to assess the associated factors with PFS. The univariate analysis provided potential $(p<0.20)$ predictive factors of PFS, with female gender $(\mathrm{HR}=0.65 \mathrm{CI} 95 \%(0.38-1.12), p=0.12)$, complete tumor resection $(\mathrm{HR}=0.19$ CI 95\% (0.09-0.37), $p<0.001$ ), complete (i.e. Mandard TRG1) pathological response $(\mathrm{HR}=0.53 \mathrm{CI} 95 \%(0.21-1.21), p=0.18)$, rectal EQD2 $\geq 45 \mathrm{~Gy}(\mathrm{HR}=0.71 \mathrm{CI} 95 \%(0.43-1.18)$, $p=0.19)$, and concomitant chemotherapy $(\mathrm{HR}=0.45 \mathrm{CI}$ $95 \%(0.18-1.13), p=0.08)$. Potential $(p<0.20)$ predictive factors of tumor progression were age $\geq 70$ years $(\mathrm{HR}=$ 2.73 CI 95\% (1.68-4.43), $p<0.001)$, low tumor location $(\mathrm{HR}=1.71 \mathrm{CI} 95 \%(1.02-2.88), p=0.04)$, time interval between radiation completion and surgery $\geq 56$ days (HR $=1.59 \mathrm{CI} 95 \%(0.53-7.0), p=0.13)$, and pre-radiation $\mathrm{NLR}>2.8(\mathrm{HR}=2.29 \mathrm{CI} 95 \%(1.35-3.90), p=0.002)$. In multivariate analysis, age $\geq 70(\mathrm{HR}=2.05 \mathrm{CI} 95 \%$ (1.18-3.54), $p=0.01)$, and pre-radiation NLR $>2.8$ (HR $=2.21 \mathrm{CI} 95 \%(1.26-3.86), p=0.006)$ were independent predictive factor of progression. Complete tumor resection $(\mathrm{HR}=0.29$ CI 95\% (0.13-0.64), $p=0.002)$ was the only independent protective factor. Uni- and multi-variate analysis is reported in Table 5.

\section{Overall Survival (OS)}

At last follow-up, 45 patients (17.5\%) had died. Median OS was therefore not reached. Two-year and 5-year OS were 91.4\% (CI 95\%: 87.9-95.1) and 77.5\% (CI 95\%: 71.5-84.1), respectively. A logistic regression (univariate and multivariate analysis) was performed in order to assess the associated factors with OS. The univariate analysis provided potential $(p<0.20)$ predictive factors of OS, with complete pathological response (HR $=0.56 \mathrm{CI} 95 \%(0.28-1.11), p=0.10)$ and concurrent chemotherapy $(\mathrm{HR}=0.48 \mathrm{CI} 95 \%(0.34-0.68), p<0.001)$. Potential $(p<0.20)$ predictive factors of death were age $\geq 70(\mathrm{HR}=3.67 \mathrm{CI} 95 \%(2.52-5.34), p<0.001), \mathrm{WHO}$ performance status $2-3(\mathrm{HR}=2.02$, CI 95\% (1.03$3.99), p=0.04)$, stage III tumors ( $\mathrm{HR}=1.51 \mathrm{CI} 95 \%$ (1.04-2.20), $p=0.03$ ), poor tumor differentiation (HR $=1.70 \mathrm{CI} 95 \%(0.81-3.60, p=0.16)$, vascular invasion $(\mathrm{HR}=1.84$ CI 95\% $(1.25-2.72), p=0.002)$, time interval between radiation completion and surgery $\geq 56$ days (HR $=1.78$ CI 95\% (1.19-2.68), $p=0.005)$ and pre-radiation NLR $>2.8(\mathrm{HR}=2.30$ CI $95 \%(1.20-4.43), p=0.01)$. In multivariate analysis, age $\geq 70(\mathrm{HR}=2.64 \mathrm{CI} 95 \%(1.35-$ $5.17), p=0.004)$, stage III tumors ( $\mathrm{HR}=2.48 \mathrm{CI} 95 \%$ (1.15-5.38), $p=0.02)$ and pre-radiation NLR $>2.8(\mathrm{HR}$ $=2.23$ CI $95 \%(1.14-2.36), p=0.02)$ were identified as independent predictive factors of death. Uni- and multivariate analysis is reported in Table 6. Overall survival depending on pre-radiation NLR is depicted in Figure 1.

\section{DISCUSSION}

Independent predictive factors of efficacy were retrospectively analyzed in a large cohort of $\mathrm{RC}$ patients in the present study, with a special focus on peripheral immune biomarkers. Pre-radiation NLR was an independent predictive factor of local relapse, of PFS, of OS and was marginally associated with complete pathological response, suggesting a value as a possible biomarker of radio-sensitivity. Combined index of peripheral neutrophil and lymphocyte counts (and especially NLR) have been shown to correlate with survival outcomes in numerous malignancies treated with definitive chemoradiation including cervical [16], lung [17] and gastrointestinal cancers [5-7, 9, 13, 14], suggesting that NLR is not specifically related to rectal (or certainly any other type of) cancer. However, the present study is one of the largest series of rectal cancer patients in literature, since numerous previous publications mixed colon and rectal cancer patients.

The NLR is thought to reflect the host' systemic inflammatory response and immunologic status, through the balance between pro- and anti-tumor activities of immune cells. On the one hand, neutrophils are suggested to induce a pro-tumor effect on the local microenvironment, secreting cytokines recruiting inflammatory cells, favoring angiogenesis [18], invasion [19], tumor growth, and suppressing the adaptive immune response [20] especially through the inhibition of natural killer cells and activated $\mathrm{T}$ cells [21]. To our best knowledge, no pre-clinical or clinical study specifically examined the underlying biological mechanisms associated with neutrophils in rectal cancer patients. However, a study was recently conducted in a large series of cervical cancer patients, resulting in appealing theories linking tumor-related neutrophilia and poor outcomes [22]. Neutrophilia (Neutrophil count $>7.5$ $\mathrm{G} / \mathrm{L}$ ) at the initial diagnosis was clearly shown to be an independent predictor of compromised survival, indeed. Furthermore, a strong correlation was observed between G-CSF immunoreactivity in patients' tumors and peripheral neutrophil count, and between G-CSF immunoreactivity and decreased survival, suggesting that the granulopoiesis induced by tumor-derived G-CSF might be the root of tumor-related neutrophilia that finally correlates with poor outcomes. Interestingly, patient outcomes were supported by strong pre-clinical data. In murine models, subjects inoculated with cancer cells expressing tumor-derived GCSF were reported to significantly develop more myeloidderived suppressor cells (a subpopulation enhancing tumor progression by stimulating tumor angiogenesis, metastasis and immune suppression) in blood, bone marrow and tumor, resulting in more aggressive tumors. Interestingly, cancer cells expressing G-GSF were significantly less sensitive to radiation tha $\mathrm{t}$ control cancer cells, through yet unclear processes [22]. On the other hand, lymphocytes are thought to have an anti-tumor effect, since it is now known for a 
Table 5: Prognostic factors for progression-free survival based on univariate and multivariate analysis $(n=257$ patients: 8 local recurrences, 38 metastatic relapses, 45 deaths)

\begin{tabular}{|c|c|c|c|c|c|}
\hline \multirow[b]{2}{*}{ Variable } & \multirow[b]{2}{*}{$\begin{array}{c}\text { Tested } v s . \text { Adverse } \\
\text { criteria }\end{array}$} & \multicolumn{2}{|c|}{ Univariate analysis } & \multicolumn{2}{|c|}{ Multivariate analysis } \\
\hline & & $\begin{array}{l}\text { Hazard ratio } \\
(95 \% \mathrm{CI})\end{array}$ & $\begin{array}{c}p \text {-value } \\
\text { (log-rank test) }\end{array}$ & $\begin{array}{l}\text { Hazard ratio } \\
(95 \% \mathrm{CI})\end{array}$ & $\begin{array}{c}p \text {-value } \\
\text { (cox model) }\end{array}$ \\
\hline Age & $\geq 70$ vs. $<70$ & $2.73(1.68-4.43)$ & $<0.001$ & $2.05(1.18-3.54)$ & 0.01 \\
\hline Gender & Female $v s$. Male & $0.65(0.38-1.12)$ & 0.12 & & \\
\hline WHO performance status & $0-1$ vs. $2-3$ & $1.23(0.30-5.04)$ & 0.77 & & \\
\hline Body mass index & $\geq 18.5$ vs. $<18.5$ & $0.60(0.14-2.47)$ & 0.48 & & \\
\hline Tumor stage & Stage III vs. I-II & $1.34(0.79-2.26)$ & 0.28 & & \\
\hline \multirow[t]{2}{*}{ Rectal tumor location } & Upper vs. Middle & $0.84(0.32-2.22)$ & 0.73 & & \\
\hline & Lower vs. Middle & $1.71(1.02-2.88)$ & 0.04 & & \\
\hline \multirow[t]{2}{*}{ Tumor differentiation } & Poor vs. Well & $0.74(0.56-1.57)$ & 0.69 & & \\
\hline & Moderate vs. Well & $0.94(0.18-3.14)$ & 0.82 & & \\
\hline Complete tumor resection & Yes vs. No & $0.19(0.09-0.37)$ & $<0.001$ & $0.29(0.13-0.64)$ & 0.002 \\
\hline ypCR & Yes vs. No & $0.53(0.21-1.32)$ & 0.18 & & \\
\hline Vascular invasion & Yes vs. No & $1.41(0.81-2.44)$ & 0.22 & & \\
\hline $\begin{array}{l}\text { Time interval between } \\
\text { radiation completion and } \\
\text { tumor resection }\end{array}$ & $\begin{array}{l}\geq 56 \text { days vs. }<56 \\
\text { days }\end{array}$ & $1.59(0.86-2.91)$ & 0.13 & & \\
\hline Pre-Radiation NLR & $>2.8 v s . \leq 2.8$ & $2.29(1.35-3.90)$ & 0.002 & $2.21(1.26-3.86)$ & 0.006 \\
\hline 4WPRN ratio & $>1.1$ vs. $\leq 1.1$ & $0.71(0.28-1.79)$ & 0.47 & & \\
\hline 4WPRL ratio & $>0.35 v s . \leq 0.35$ & $1.42(0.75-2.68)$ & 0.27 & & \\
\hline 4WPL ratio & $>2.5 v s . \leq 2.5$ & $1.20(0.59-2.45)$ & 0.61 & & \\
\hline \multirow[t]{3}{*}{$\begin{array}{l}\text { Radiotherapy } \\
\text { characteristics }\end{array}$} & $\begin{array}{l}\text { Rectal EQD2 } \geq 45 \\
\text { Gy } v s .<45 \text { Gy }\end{array}$ & $0.71(0.43-1.18)$ & 0.19 & $0.61(0.35-1.08)$ & 0.09 \\
\hline & $\begin{array}{l}\text { Pelvis EQD2 } \geq 40 \\
\text { Gy vs. }<40 \text { Gy }\end{array}$ & $1.35(0.33-5.55)$ & 0.67 & & \\
\hline & $\begin{array}{l}\text { Hypofractionated } v s . \\
\text { Normofractionated }\end{array}$ & $1.44(0.65-3.15)$ & 0.36 & & \\
\hline $\begin{array}{l}\text { Concomitant } \\
\text { chemotherapy }\end{array}$ & Yes $v s$. No & $0.45(0.18-1.13)$ & 0.09 & & \\
\hline
\end{tabular}

95\% CI: 95\% confidence interval; ypCR: Pathological complete response (Mandard TRG1); NLR: Neutrophil to Lymphocyte ratio; 4WPRN ratio: "4 weeks" to "pre-radiation" neutrophil ratio; 4WPRL ratio: "4 weeks" to "pre-radiation" lymphocyte ratio; 4WPL ratio: "4 weeks" neutrophil to "4 weeks" lymphocyte ratio. N.A.: Not assessable (no event), typically giving infinity values. EQD2: Equivalent Dose in 2 Grays per fraction; Normofractionated: $<2.5$ Gy per fraction; Hypofractionated: $\geq 2.5$ Gy per fraction.

All $p$-values $\leq 0.2$ in univariate analysis have been tested in multivariate analysis, except correlated variables (correlation with $p<0.001$ ). Finally, only bold typed values were tested in multivariate analysis.

long time that tumor infiltration of CD4+ and CD8+ cells is associated with improved survival in RC patients [23, 24]. Furthermore, lymphocytes are suggested to play a crucial role in cancer immune surveillance and immunoediting [25], probably explaining why patients with high lymphocyte densities achieve long term local and distant control after resection [23]. Elevated NLR could therefore reflect the predominance of neutrophils' pro-tumor activity and/or the weakness of the lymphocytes' antitumor immune response. The exact biological phenomena underlying the fact that patients with elevated NLR show unfavorable local and overall outcome are still investigated, but taken together, all results seem to indicate that the NLR is a simple and robust biomarker for risk stratification in locally advanced RC patients, with a threshold ranging from 2 to 5 in literature [26, 27]. Although the relation 
Table 6: Prognostic factors for Overall Survival based on univariate and multivariate analysis $(n=257$ patients with 45 deaths)

\begin{tabular}{|c|c|c|c|c|c|}
\hline \multirow[b]{2}{*}{ Variable } & \multirow[b]{2}{*}{$\begin{array}{c}\text { Tested vs. Adverse } \\
\text { criteria }\end{array}$} & \multicolumn{2}{|c|}{ Univariate analysis } & \multicolumn{2}{|c|}{ Multivariate analysis } \\
\hline & & $\begin{array}{c}\text { Hazard ratio } \\
(95 \% \mathrm{CI})\end{array}$ & $\begin{array}{c}p \text {-value } \\
\text { (log-rank test) }\end{array}$ & $\begin{array}{c}\text { Hazard ratio } \\
(95 \% \mathrm{CI})\end{array}$ & $\begin{array}{c}p \text {-value } \\
\text { (cox model) }\end{array}$ \\
\hline Age & $\geq 70$ vs. $<70$ & $3.67(2.52-5.34)$ & $<0.001$ & $2.64(1.35-5.17)$ & 0.004 \\
\hline Gender & Female vs. Male & $0.81(0.57-1.17)$ & 0.27 & & \\
\hline WHO performance status & $2-3$ vs. $0-1$ & $2.02(1.03-3.99)$ & 0.04 & & \\
\hline Body mass index & $\geq 18.5$ vs. $<18.5$ & $0.74(0.30-1.83)$ & 0.52 & & \\
\hline Tumor stage & Stage III vs. I-II & $1.51(1.04-2.20)$ & 0.03 & $2.48(1.15-5.38)$ & 0.02 \\
\hline \multirow[t]{2}{*}{ Rectal tumor location } & Upper vs. Middle & $1.13(0.65-1.96)$ & 0.66 & & \\
\hline & Lower vs. Middle & $1.19(0.82-1.73)$ & 0.35 & & \\
\hline \multirow[t]{2}{*}{ Tumor differentiation } & Poor vs. Well & $1.70(0.81-3.60)$ & 0.16 & & \\
\hline & Moderate vs. Well & $1.10(0.75-1.60)$ & 0.64 & & \\
\hline $\begin{array}{l}\text { Complete tumor } \\
\text { resection }\end{array}$ & Yes vs. No & $0.33(0.21-0.53)$ & $<0.001$ & & \\
\hline ypCR & Yes vs. No & $0.56(0.28-1.11)$ & 0.10 & & \\
\hline Vascular invasion & Yes vs. No & $1.84(1.25-2.72)$ & 0.002 & & \\
\hline $\begin{array}{l}\text { Time interval between } \\
\text { RT completion and } \\
\text { tumor resection }\end{array}$ & $\begin{array}{l}\geq 56 \text { days vs. }<56 \\
\text { days }\end{array}$ & $1.78(1.19-2.68)$ & 0.005 & & \\
\hline Pre-Radiation NLR & $>2.8 v s . \leq 2.8$ & $2.30(1.20-4.43)$ & 0.01 & $2.23(1.14-2.36)$ & 0.02 \\
\hline $4 \mathrm{WPRN}$ ratio & $>1.1 \mathrm{vs} \leq 1.1$ & $0.78(0.27-2.21)$ & 0.64 & & \\
\hline 4WPRL ratio & $>0.35$ vs $\leq 0.35$ & $1.09(0.52-2.29)$ & 0.81 & & \\
\hline 4WPL ratio & $>2.5 \mathrm{vs} \leq 2.5$ & $1.5(0.63-3.57)$ & 0.363 & & \\
\hline \multirow[t]{3}{*}{$\begin{array}{l}\text { Radiotherapy } \\
\text { characteristics }\end{array}$} & $\begin{array}{l}\text { Rectal EQD2 } \geq 45 \\
\text { Gy vs. }<45 \text { Gy }\end{array}$ & $0.99(0.69-1.43)$ & 0.97 & & \\
\hline & $\begin{array}{l}\text { Pelvis EQD2 } \geq 40 \\
\text { Gy vs. }<40 \mathrm{~Gy}\end{array}$ & $0.94(0.56-1.57)$ & 0.81 & & \\
\hline & $\begin{array}{l}\text { Hypofractionated } v s \text {. } \\
\text { Normaofractionated }\end{array}$ & $1.00(0.58-1.70)$ & 0.99 & & \\
\hline $\begin{array}{l}\text { Concomitant } \\
\text { chemotherapy }\end{array}$ & Yes vs. No & $0.48(0.34-0.68)$ & $<0.001$ & & \\
\hline
\end{tabular}

95\% CI: 95\% confidence interval; ypCR: Pathological complete response (Mandard TRG1); NLR: Neutrophil to Lymphocyte ratio; 4WPRN ratio: "4 weeks" to "pre-radiation" neutrophil ratio; 4WPRL ratio: "4 weeks" to "pre-radiation" lymphocyte ratio; 4WPL ratio: "4 weeks" neutrophil to "4 weeks" lymphocyte ratio. N.A.: Not assessable (no event), typically giving infinity values. EQD2: Equivalent Dose in 2 Grays per fraction; Normofractionated: $<2.5$ Gy per fraction; Hypofractionated: $\geq 2.5$ Gy per fraction.

All $p$-values $\leq 0.2$ in univariate analysis have been tested in multivariate analysis, except correlated variables (correlation with $p<0.001)$. Finally, only bold typed values were tested in multivariate analysis.

between peripheral immune biomarkers and radiation sensitivity is not yet established, the influence of tumor micro-environment and radiosensitivity has been clearly demonstrated [28-30]. The present findings support the hypothesis that immune cell counts, which presumably reflect host conditions, may critically affect responsiveness to radiation.

These results are of primary interest since conventional prognostic factors currently fail to identify patients with the highest risk of local and metastatic 
recurrence, with more than $40 \%$ of patients experiencing lethal relapse. The present findings could therefore contribute to isolate a subpopulation eligible for treatment intensification (radiation dose escalation and/ or chemotherapy optimization) at the outset. The current standard of care for locally advanced RC is a neoadjuvant chemoradiotherapy [31, 32] followed by definitive resection. A post-operative chemotherapy is indicated only when bad prognosis factors are identified after rectal surgery, inducing favorable results on local recurrence and PFS [1] but disappointing results on OS [1, 33-35]. Post-operative chemotherapy failed to improve OS in all randomized trials using pre-operative chemoradiation followed by total mesorectal excision, indeed. However, less than $50 \%$ of eligible patients received the full dose and course without interruption or delays owing to postoperative complications, delayed recovery, or interference caused by ostomy closure. For the same reasons, nearly a third of patients with locally advanced RC never started the indicated adjuvant chemotherapy. In the present study, similar orders of magnitude were reported, with more than a patient out of ten not undergoing the indicated adjuvant chemotherapy, and with about on patient out of two who could not receive the full dose. Therefore, moving the setting of chemotherapy from adjuvant to neoadjuvant is carefully considered, since it is expected to bring a better safety (better bone marrow function, no stoma resulting in less treatment delays and dose reduction because of stoma complications, shorter duration of stoma use) and maybe a better efficacy (early treatment of the micrometastatic disease, better local response making the surgery unnecessary in selected patients). Finally, there is growing evidence suggesting that patients with bad prognosis factors could benefit from a neoadjuvant chemotherapy performed before chemoradiation [36, 37]. Currently known factors of high risk of distant relapse (node involvement, mesorectal invasion, positive circumferential resection margin, advanced $\mathrm{T}$ stage) were recently hypothesized to possibly indicate a preoperative chemotherapy. Clinical trials are currently conducted to explore the efficacy of this management, through the RAPIDO (NCT01558921), PROSPECT (NCT01515787), NRGGI002 (NCT02921256), BACCHUS (NCT01650428) and Rectal Cancer Consortium (NCT02008656) clinical trials. However, since current RC prognosis factors are thought to be not discriminant enough, and since many of them are only available after surgery completion or state-of-the-art MRI, new simple pre-treatment biomarkers are awaited in order to better predict which patients will be non-responders to chemoradiation, and should therefore experience neo-

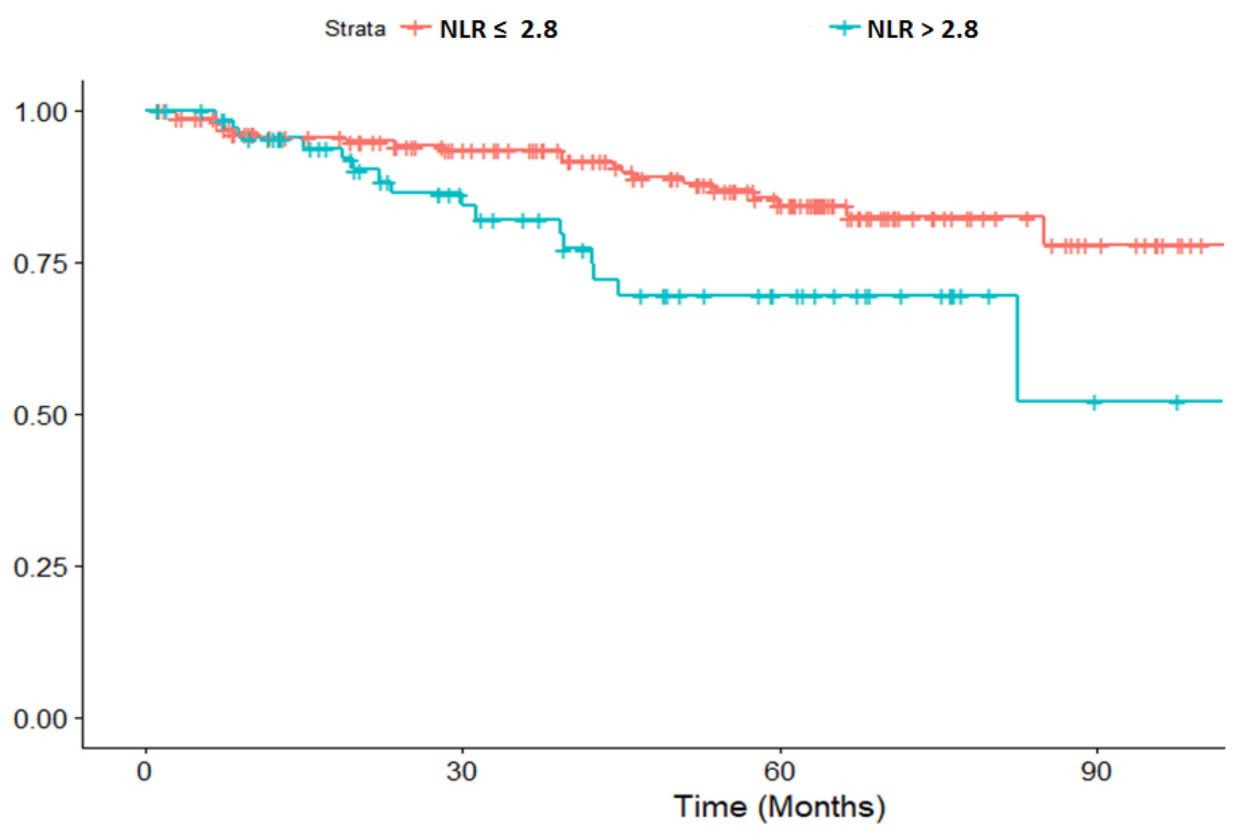

\begin{tabular}{|c|c|c|c|c|}
\hline \multicolumn{5}{|c|}{ Number at risk } \\
\hline$N L R \leq 2.8$ & 169 & 122 & 68 & 12 \\
\hline NLR $>2.8$ & 71 & 40 & 18 & 2 \\
\hline & 0 & 30 & $\begin{array}{r}60 \\
\text { (M }\end{array}$ & 90 \\
\hline
\end{tabular}

Figure 1: Overall survival depending on the pre-radiation Neutrophil to Lymphocyte Ratio (NLR). (HR 2.23 CI 95\% $(\mathbf{1 . 1 4}-2.36), p=0.02)$ 
adjuvant chemotherapy. In this context, the pre-treatment NLR seem to be able to bring crucial information on prognostication before treatment initiation, being an independent predictive factor of local recurrence, PFS and OS. Although the retrospective nature of the present study is a limitation, these results should encourage the prospective exploration of peripheral immune biomarkers in $\mathrm{RC}$ patients, in order to validate its metrological characteristics.

\section{MATERIALS AND METHODS}

A retrospective study was conducted at the Lucien Neuwirth comprehensive cancer care center (Saint Priest en Jarez, France). The institutional review board approved the study, which was conducted in compliance with the Helsinki Declaration.

\section{Patient population and biology}

Medical records of all consecutive patients undergoing a pre-operative radiotherapy for a nonmetastatic RC between 2004 and 2015 were retrospectively reviewed. Only patients with available biological data (i.e. neutrophil and/or lymphocyte counts) were selected. Patient characteristics (age, sex, WHO performance status, body mass index (BMI)), tumor histology and staging, radiotherapy characteristics, administered chemotherapy, complete sterilization of the operative specimen (ypCR), complete tumor resection (R0), local recurrence, progression-free survival (PFS) and overall survival (OS) were also studied. Regarding pathological response, the Mandard tumor regression grade (TRG) was also used [38], classifying patients into "good responders" (complete pathological response with no viable cancer cell (TRG1, i.e. $\mathrm{ypCR}$ ) or almost complete pathological response with rare cancer cells that might be still viable (TRG2)) and into "poor responders" (Mandard TGR3-5).

The absolute neutrophil and lymphocyte counts were obtained from samples collected within 30 days prior to radiotherapy initiation and during the fourth week after radiation initiation. Neutrophil to Lymphocyte Ratio (NLR) was obtained by dividing absolute pre-radiation neutrophil to lymphocyte count. "Four-weeks" to "preradiation" neutrophil (4WPRN) ratio was obtained by dividing absolute 4-weeks neutrophil count to preradiation neutrophil count. "Four-weeks" to "pre-radiation" lymphocyte (4WPRL) ratio was obtained by dividing absolute 4-weeks lymphocyte count to pre-radiation lymphocyte count. "Four weeks" neutrophil to lymphocyte ratio (4WPL) was obtained by dividing absolute 4-week neutrophil count to 4-weeks lymphocyte count. The Contal and Q'Quigley method was used to find optimal threshold values for NLR, 4WPRN, 4WPRL and 4WPL. In the whole set of patient, a NLR cut-off of 2.8 was found to have the highest log-rank statistic (regarding OS). For
4WPRN, 4WPRL and 4WPL ratios, cut-offs points of 1.1, 0.35 and 2.5 were identified, respectively.

\section{Treatment definition}

\section{Radiation therapy}

Patients were treated in supine position, and immobilized using leg-positioning foamed wedges. CTscan images were acquired without contrast agent infusion with a slice thickness of $2.5 \mathrm{~mm}$. Plans were contoured and calculated using the Eclipse treatment planning system (Varian Medical Systems, Palto Alto, CA, USA). Gross tumor volume (GTV), clinical tumor volume (CTV), planning tumor volume (PTV) and organs at risk (OAR) were delineated based on planning-CT. Their definition evolved with the availability and development of CT-scan and MRI, and with the delineation guidelines' editions. In each case, treatment plans were optimized according to dose limits for OAR and constraints for volume coverage i.e. PTV should receive $95 \%$ to $107 \%$ of the prescribed dose. Rectal equivalent 2 Gy (EQD2) dose was calculated using the EQD2 formula provided by Fowler [39] and $\alpha / \beta$ $=6.2[40]$.

\section{Surgery}

A curative rectal resection (Total Mesorectal Excision) was systematically performed after radiotherapy completion.

\section{Evaluation of efficacy}

Follow-up was calculated from the initiation of radiotherapy. After radiotherapy completion, patients were assessed for efficacy every 3 months by surgeons and oncologists during the first two years and every 6 months later, with clinical examination and alternation of chest/abdomen/pelvis- CT-scan and chest radiography and abdominal ultrasound.

\section{Statistical analysis}

Median values were given with the interquartile range (IQR: $25 \%-75 \%$ ) or with the range (Min-Max). Chi2 test, Kruskal-Wallis test or Fisher test were performed to compare patient characteristics distribution. PFS was defined as the time from the date of radiotherapy initiation to the date of clinical and/or radiological RC progression. OS was defined as the time from the date of radiotherapy initiation to the date of death or the last follow-up. PFS and OS were estimated with the Kaplan-Meier method. Median survivals were compared using log-rank test. A Cox proportional hazards model was used to test the interaction between data on survival or on local control and treatment or patient characteristics. The multivariate 
analysis was performed using a Cox multivariate analysis based on the significant -or close-to-significance $(p<0.2)$ factors. The multivariate model was refined using the AIC criteria. All $p$ values were nominal without adjustment for multiple testing. Significance was defined by $p<0.05$. Statistical analyses were processed with R-3•4•0 (R Core Team. R Foundation for Statistical Computing, Vienna, Austria).

\section{CONCLUSIONS}

Pre-radiation NLR is a simple and robust biomarker for risk stratification in locally advanced $\mathrm{RC}$ patients undergoing pre-operative radiotherapy. Indeed, it was correlated with local recurrence, PFS and OS in multivariate analysis. NLR was marginally associated with complete pathological response, suggesting a value as a possible biomarker of radio-sensitivity. These results could lead the way to the accurate selection of the subpopulation of RC patients eligible to neoadjuvant chemotherapy. Although NLR is not a quite new marker, with more than 100 studies on colorectal adenocarcinoma available in MEDLINE, the present data corroborates previous results, in one of the largest cohort of RC Caucasian patients treated with pre-TME radiation. A study examining the underlying biological mechanisms connecting LNR with poor prognosis and local radioresistance in RC patients is still to be carried out. Finally, the retrospective nature of the study induced unavoidable statistical biases that could only be controlled by prospective explorations. Therefore, statistical correlations that were presently suggested should be corroborated by future prospective studies.

\section{Abbreviations}

AIC: Akaike Information Criteria; BMI: Body Mass Index; HR: Hazard Ratio; IQR: Interquartile Range; NLR: Neutrophil to Lymphocyte Ratio; MRI: Magnetic Resonance Imaging; OS: Overall Survival; PFS: Progression-Free Survival; RC: Rectal Cancer; R0: complete tumor regression; TRG: Tumor Regression Grade; ypCR: complete sterilization of the operative specimen; WHO: World Health Organization; 4WPRN ratio: "Four-weeks" to "pre-radiation" neutrophil ratio; 4WPRL ratio: "Four-weeks" to "pre-radiation" lymphocyte ratio; 4WPL ratio: "Four weeks" neutrophil to lymphocyte ratio.

\section{Author contributions}

NM, JLE, AV, MAG, PD: conception and design of the study; MBM, DK, TM, GP, AV, JLE, PD, MAG: literature review, data management and analysis; SE, GDL, JBG, JP, JMP, AV, JLE, NM, MP : drafting, critical revision and editing. All authors approved the final version.

\section{ACKNOWLEDGMENTS}

Jeffrey Perkins for his English language support.

\section{CONFLICTS OF INTEREST}

Authots declare no conflicts of interest.

\section{FUNDING}

None.

\section{REFERENCES}

1. Breugom AJ, Swets M, Bosset JF, Collette L, Sainato A, Cionini L, Glynne-Jones R, Counsell N, Bastiaannet E, van den Broek CB, Liefers GJ, Putter H, van de Velde CJ. Adjuvant chemotherapy after preoperative (chemo) radiotherapy and surgery for patients with rectal cancer: a systematic review and meta-analysis of individual patient data. Lancet Oncol. 2015; 16:200-07.

2. Compton CC, Fielding LP, Burgart LJ, Conley B, Cooper HS, Hamilton SR, Hammond ME, Henson DE, Hutter RV, Nagle RB, Nielsen ML, Sargent DJ, Taylor CR, et al. Prognostic factors in colorectal cancer. College of American Pathologists Consensus Statement 1999. Arch Pathol Lab Med. 2000; 124:979-94.

3. Tie J, Cohen J, Wang Y, Li L, Kinde I, Elsaleh H, Wong R, Kosmider S, Yip D, Lee M, Tran B, Burge ME, Goldstein $\mathrm{D}$, et al. The potential of circulating tumor DNA (ctDNA) to guide adjuvant chemotherapy decision making in locally advanced rectal cancer (LARC). J Clin Oncol. 2017; 35:3521.

4. Chopra N, Turner NC. Targeting PIK3CA-mutant advanced breast cancer in the clinical setting. Lancet Oncol. 2017; 18:842-43.

5. Schernberg A, Escande A, Rivin Del Campo E, Ducreux M, Nguyen F, Goere D, Chargari C, Deutsch E. Leukocytosis and neutrophilia predicts outcome in anal cancer. Radiother Oncol. 2017; 122:137-45.

6. Schernberg A, Moureau-Zabotto L, Rivin Del Campo E, Escande A, Ducreux M, Nguyen F, Goere D, Chargari C, Deutsch E. Leukocytosis and neutrophilia predict outcome in locally advanced esophageal cancer treated with definitive chemoradiation. Oncotarget. 2017; 8:1157911588. https://doi.org/10.18632/oncotarget.14584.

7. Schernberg A, Huguet F, Moureau-Zabotto L, Chargari C, Rivin Del Campo E, Schlienger M, Escande A, Touboul E, Deutsch E. External validation of leukocytosis and neutrophilia as a prognostic marker in anal carcinoma treated with definitive chemoradiation. Radiother Oncol. 2017; 124:110-17.

8. Sumner WA, Stokes WA, Oweida A, Berggren KL, McDermott JD, Raben D, Abbott D, Jones B, Gan G, Karam SD. Survival impact of pre-treatment neutrophils 
on oropharyngeal and laryngeal cancer patients undergoing definitive radiotherapy. J Transl Med. 2017; 15:168.

9. Galizia G, Lieto E, Zamboli A, De Vita F, Castellano P, Romano C, Auricchio A, Cardella F, De Stefano L, Orditura M. Neutrophil to lymphocyte ratio is a strong predictor of tumor recurrence in early colon cancers: A propensity scorematched analysis. Surgery. 2015; 158:112-20.

10. Absenger G, Szkandera J, Pichler M, Stotz M, Arminger F, Weissmueller M, Schaberl-Moser R, Samonigg H, Stojakovic T, Gerger A. A derived neutrophil to lymphocyte ratio predicts clinical outcome in stage II and III colon cancer patients. Br J Cancer. 2013; 109:395-400.

11. Sung S, Son SH, Park EY, Kay CS. Prognosis of locally advanced rectal cancer can be predicted more accurately using pre- and post-chemoradiotherapy neutrophillymphocyte ratios in patients who received preoperative chemoradiotherapy. PLoS One. 2017; 12:e173955.

12. Caputo D, Caricato M, Coppola A, La Vaccara V, Fiore M, Coppola R. Neutrophil to Lymphocyte Ratio (NLR) and Derived Neutrophil to Lymphocyte Ratio (d-NLR) Predict Non-Responders and Postoperative Complications in Patients Undergoing Radical Surgery After Neo-Adjuvant Radio-Chemotherapy for Rectal Adenocarcinoma. Cancer Invest. 2016:1-12.

13. Kim IY, You SH, Kim YW. Neutrophil-lymphocyte ratio predicts pathologic tumor response and survival after preoperative chemoradiation for rectal cancer. BMC Surg. 2014; $14: 94$.

14. Shen L, Zhang H, Liang L, Li G, Fan M, Wu Y, Zhu J, Zhang Z. Baseline neutrophil-lymphocyte ratio $(\geq 2.8)$ as a prognostic factor for patients with locally advanced rectal cancer undergoing neoadjuvant chemoradiation. Radiat Oncol. 2014; 9:295.

15. Nagasaki T, Akiyoshi T, Fujimoto Y, Konishi T, Nagayama S, Fukunaga Y, Ueno M. Prognostic Impact of Neutrophilto-Lymphocyte Ratio in Patients with Advanced Low Rectal Cancer Treated with Preoperative Chemoradiotherapy. Dig Surg. 2015; 32:496-503.

16. Escande A, Haie-Meder C, Maroun P, Gouy S, Mazeron R, Leroy T, Bentivegna E, Morice P, Deutsch E, Chargari C. Neutrophilia in locally advanced cervical cancer: A novel biomarker for image-guided adaptive brachytherapy? Oncotarget. 2016; 7:74886-74894. https://doi.org/10.18632/ oncotarget. 12440 .

17. Käsmann L, Bolm L, Schild SE, Janssen S, Rades D. Neutrophil-to-Lymphocyte Ratio Predicts Outcome in Limited Disease Small-cell Lung Cancer. Hai. 2017; 195:217-24.

18. Kusumanto YH, Dam WA, Hospers GA, Meijer C, Mulder NH. Platelets and granulocytes, in particular the neutrophils, form important compartments for circulating vascular endothelial growth factor. Angiogenesis. 2003; 6:283-87.

19. Shamamian P, Schwartz JD, Pocock BJ, Monea S, Whiting D, Marcus SG, Mignatti P. Activation of progelatinase A (MMP-2) by neutrophil elastase, cathepsin G, and proteinase-3: a role for inflammatory cells in tumor invasion and angiogenesis. J Cell Physiol. 2001; 189:197-206.

20. Fridlender ZG, Albelda SM. Tumor-associated neutrophils: friend or foe? Carcinogenesis. 2012; 33:949-55.

21. Di Carlo E, Forni G, Musiani P. Neutrophils in the antitumoral immune response. Chem Immunol Allergy. 2003; 83:182-203.

22. Mabuchi S, Matsumoto Y, Kawano M, Minami K, Seo Y, Sasano T, Takahashi R, Kuroda H, Hisamatsu T, Kakigano A, Hayashi M, Sawada K, Hamasaki T, et al. Uterine cervical cancer displaying tumor-related leukocytosis: a distinct clinical entity with radioresistant feature. J Natl Cancer Inst. 2014; 106:dju147.

23. Fridman WH, Pagès F, Sautès-Fridman C, Galon J. The immune contexture in human tumours: impact on clinical outcome. Nat Rev Cancer. 2012; 12:298-306.

24. Jass JR. Lymphocytic infiltration and survival in rectal cancer. J Clin Pathol. 1986; 39:585-89.

25. Dunn GP, Old LJ, Schreiber RD. The immunobiology of cancer immunosurveillance and immunoediting. Immunity. 2004; 21:137-48.

26. Mei Z, Shi L, Wang B, Yang J, Xiao Z, Du P, Wang Q, Yang W. Prognostic role of pretreatment blood neutrophilto-lymphocyte ratio in advanced cancer survivors: A systematic review and meta-analysis of 66 cohort studies. Cancer Treat Rev. 2017; 58:1-13.

27. Li MX, Liu XM, Zhang XF, Zhang JF, Wang WL, Zhu Y, Dong J, Cheng JW, Liu ZW, Ma L, Lv Y. Prognostic role of neutrophil-to-lymphocyte ratio in colorectal cancer: a systematic review and meta-analysis. Int J Cancer. 2014; 134:2403-13.

28. Vallard A, Espenel S, Guy JB, Diao P, Xia Y, El Meddeb Hamrouni A, Ben Mrad M, Falk AT, Rodriguez-Lafrasse C, Rancoule C, Magné N. Targeting stem cells by radiation: from the biological angle to clinical aspects. World J Stem Cells. 2016; 8:243-50.

29. Barcellos-Hoff MH, Park C, Wright EG. Radiation and the microenvironment-tumorigenesis and therapy. Nat Rev Cancer. 2005; 5:867-75.

30. Prise KM, Schettino G, Folkard M, Held KD. New insights on cell death from radiation exposure. Lancet Oncol. 2005; 6:520-28.

31. Sineshaw HM, Jemal A, Thomas CR Jr, Mitin T. Changes in treatment patterns for patients with locally advanced rectal cancer in the United States over the past decade: An analysis from the National Cancer Data Base. Cancer. 2016; 122:1996-2003.

32. Cassidy RJ, Liu Y, Patel K, Zhong J, Steuer CE, Kooby DA, Russell MC, Gillespie TW, Landry JC. Can we eliminate neoadjuvant chemoradiotherapy in favor of neoadjuvant multiagent chemotherapy for select stage II/III rectal adenocarcinomas: Analysis of the National Cancer Data base. Cancer. 2017; 123:783-93. 
33. Lutz MP, Zalcberg JR, Glynne-Jones R, Ruers T, Ducreux M, Arnold D, Aust D, Brown G, Bujko K, Cunningham C, Evrard S, Folprecht G, Gerard JP, et al. Second St. Gallen European Organisation for Research and Treatment of Cancer Gastrointestinal Cancer Conference: consensus recommendations on controversial issues in the primary treatment of rectal cancer. Eur J Cancer. 2016; 63:11-24.

34. Bujko K, Glimelius B, Valentini V, Michalski W, Spalek M. Postoperative chemotherapy in patients with rectal cancer receiving preoperative radio(chemo)therapy: A meta-analysis of randomized trials comparing surgery \pm a fluoropyrimidine and surgery + a fluoropyrimidine \pm oxaliplatin. Eur J Surg Oncol. 2015; 41:713-23.

35. Petersen SH, Harling H, Kirkeby LT, Wille-Jørgensen P, Mocellin S. Postoperative adjuvant chemotherapy in rectal cancer operated for cure. Cochrane Database Syst Rev. 2012; CD004078:CD004078.

36. Fernández-Martos C, Pericay C, Aparicio J, Salud A, Safont M, Massuti B, Vera R, Escudero P, Maurel J, Marcuello E, Mengual JL, Saigi E, Estevan R, et al. Phase II, randomized study of concomitant chemoradiotherapy followed by surgery and adjuvant capecitabine plus oxaliplatin (CAPOX) compared with induction CAPOX followed by concomitant chemoradiotherapy and surgery in magnetic resonance imaging-defined, locally advanced rectal cancer: grupo cancer de recto 3 study. J Clin Oncol. 2010; 28:859-65.

37. Cercek A, Goodman KA, Hajj C, Weisberger E, Segal NH, Reidy-Lagunes DL, Stadler ZK, Wu AJ, Weiser MR, Paty PB, Guillem JG, Nash GM, Temple LK, et al. Neoadjuvant chemotherapy first, followed by chemoradiation and then surgery, in the management of locally advanced rectal cancer. J Natl Compr Canc Netw. 2014; 12:513-19.

38. Santos MD, Silva C, Rocha A, Nogueira C, CastroPoças F, Araujo A, Matos E, Pereira C, Medeiros R, Lopes C. Predictive clinical model of tumor response after chemoradiation in rectal cancer. Oncotarget. 2017; 8:58133-51. https://doi.org/10.18632/oncotarget.19651.

39. Fowler JF. Sensitivity analysis of parameters in linearquadratic radiobiologic modeling. Int J Radiat Oncol Biol Phys. 2009; 73:1532-37.

40. Nahum AE. The radiobiology of hypofractionation. Clin Oncol (R Coll Radiol). 2015; 27:260-69. 